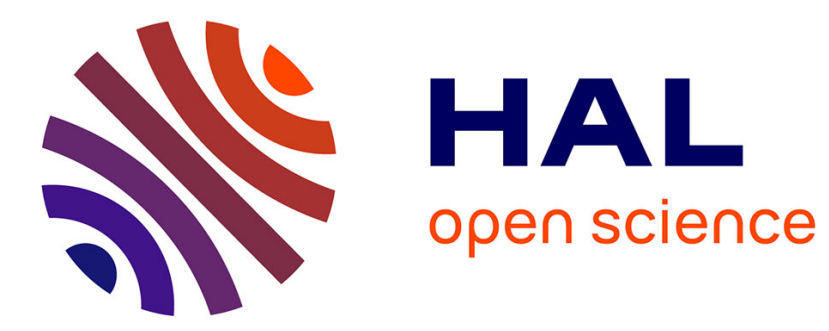

\title{
Characterizing mechanical change in metals using amplitude-modulated diffuse ultrasound
}

Fan Xie, Yuxiang Zhang, Éric Larose, Aroune Duclos, Su Chen, Xiaoyun Li, Chunlong Fei

\section{- To cite this version:}

Fan Xie, Yuxiang Zhang, Éric Larose, Aroune Duclos, Su Chen, et al.. Characterizing mechanical change in metals using amplitude-modulated diffuse ultrasound. Structural Health Monitoring, 2020, pp.147592172090504. 10.1177/1475921720905046 . hal-02503075

\section{HAL Id: hal-02503075 \\ https://hal.science/hal-02503075}

Submitted on 20 Oct 2021

HAL is a multi-disciplinary open access archive for the deposit and dissemination of scientific research documents, whether they are published or not. The documents may come from teaching and research institutions in France or abroad, or from public or private research centers.
L'archive ouverte pluridisciplinaire HAL, est destinée au dépôt et à la diffusion de documents scientifiques de niveau recherche, publiés ou non, émanant des établissements d'enseignement et de recherche français ou étrangers, des laboratoires publics ou privés. 


\title{
Characterizing mechanical change in metals using amplitude- modulated diffuse ultrasound
}

Fan Xie ${ }^{1}$, Yuxiang Zhang ${ }^{2}$, Eric Larose ${ }^{3}$, Aroune Duclos ${ }^{4}, \mathrm{Su} \mathrm{Chen}^{1}$, Xiaoyun $\mathrm{Li}^{5}$, Chunlong $\mathrm{Fei}^{6}$

\begin{abstract}
In this paper, we present an ultrasonic method based on diffuse ultrasound with successive excitation amplitudes. The system provides amplitude-dependent parameters of diffuse ultrasound using coda wave interferometry, and can be used to characterize mechanical change in metallic materials. Localized mechanical change caused by instantaneous $400{ }^{\circ} \mathrm{C}$ thermal shocking in a meter-scale aluminum alloy slab were successfully characterized using variations in velocity and decorrelation coefficients with excitation amplitudes. We discuss the mechanisms and spatial distribution potentially causing the observed amplitudedependent diffuse waveform modification. Combining the method presented here with complementary approaches will enhance the ability to nondestructively detect early-stage damage in metals either in the laboratory or for on-site applications.
\end{abstract}

\section{Keywords}

diffuse ultrasound, nonlinear effect, coda wave interferometry, amplitude-modulation, aluminum alloy, mechanical change

\section{Introduction}

Due to their direct sensitivity to the elastic properties of materials along with their costeffective and reliable performance, ultrasound- based Non-Destructive Testing (NDT) techniques provide a primary tool to assess the condition, integrity and reliability of a wide range of metals, and to monitor for potential risks throughout their life cycle. In recent years, research has sought techniques to detect the initiation of damage as early as possible, playing a key role in the development of NDT approaches. Indeed, mechanical changes which cause damage in its very early stages may jeopardize the normal operation of a material. It is therefore necessary to develop more accurate and sensitive ultrasound-based techniques to assess material structures.

Among the emerging techniques, nonlinear diffuse ultrasound shows great promise since the scattered waves are more sensitive to the nonlinear elastic response of the material than direct 
waves. Consequently, it provides information that is complementary to that provided by common bulk nonlinear techniques when performing global/local inspection of a material's integrity at the early stages of damage. For instance, depending on the quasi-static acoustoelastic effect ${ }^{1}$, high-order nonlinear elastic constants can be determined by monitoring velocity changes in diffuse waves at different times thanks to coda wave interferometry (CWI) performed during very small incremental stress solicitations (down to a few $\mathrm{kPa}$ ) ${ }^{2,3}$. A particularly attractive nonlinear elastic effect, time-logarithmic recovery (referred to as slow dynamics) back to the unperturbed elastic modulus following a sharp drop in the elastic modulus (referred to as fast dynamics) has been reported in response to moderate mechanical solicitations ${ }^{4,5}$. Such nonlinear responses were first probed with nonlinear resonant ultrasound spectroscopy (NRUS) ${ }^{6}$, and it can also be monitored efficiently with velocity changes from CWI.

More recently, several authors ${ }^{7-9}$ proposed an alternative nonlinear "pump-probe" method consisting in measuring low-amplitude diffuse waves at high frequencies (known as the HF probe wave) by CWI to detect the elastic changes induced by another large-amplitude wave excitation at a lower frequency (LF pump wave). In these experiments, both excitations were performed simultaneously, with the probe wave (CWI) triggered several times during the pump excitation. This pump excitation is thought to activate nonlinear behavior in cracks present in an initially linear elastic medium, thus altering the material's elasticity. This technique can therefore assess overall damage levels by quantifying CWI versus the pump level, which derived from the so-called "quadratic hysteretic nonlinearity". The nonlinear response depends on the presence of micro cracks or soft inhomogeneities that act as nonlinear sources ${ }^{10,11}$. Recent developments ${ }^{9,11,15}$ show that the relative wave velocity change being linearly proportional to the pump wave amplitude, meanwhile the pump wave amplitude also leads to a quadratic increase in decorrelation $(\mathrm{kd})$.

Nevertheless, this type of monitoring remains challenging from the NDT point of view because the resolution is much larger than that of the micro cracks: at least several tens of wavelengths in a heterogeneous medium ${ }^{9,12}$. Locating the change thus becomes extremely challenging. In addition, nonlinear diffuse wave techniques are less sensitive in homogeneous metals where changes induced by micro cracks only become detectable at a scale of a dozen of microns. In contrast, diffuse ultrasound is sensitive to changes in environmental (e.g. temperature, humidity etc. $)^{13,14}$ and operational conditions (e.g. the a priori reference state, external mechanical loading or excitation facilities), that are difficult to measure in normal 
NDT operation. Consequently, most current diffuse wave- based nonlinear ultrasound techniques only work in laboratory conditions, where damage detection resolution and the dimensions of the test specimen are both limited ${ }^{8,15,16}$.

Aluminum alloys are widely used in load-bearing applications (e.g. bridge decks, marine crafts, nuclear power engineering etc.). Aluminum is more subject to the alteration of mechanical properties following a fire as the phase transition temperatures for this material are significantly lower than that of steel ${ }^{17,18}$. However, little research on damage has been done to assess the ability to relate changes in mechanical properties to microstructural alterations following an exposure to an elevated temperate ${ }^{19}$.

In this article, we present a simple and robust high-frequency (HF) diffuse ultrasound-based method with successive excitation amplitudes, which expands to the early damage detection capabilities of the method. Nonlinear diffuse ultrasound is used to provide amplitudedependent parameters using CWI. Our goal is to quickly assess changes in mechanical properties at an early-stage damage in metals.

\section{Materials and methods}

\section{Aluminum alloy sample}

The tests were conducted on an intact $20 \mathrm{~mm}$ thick aluminum alloy slab (6061) measuring $1000 \mathrm{~mm} \times 1000 \mathrm{~mm}$. The chemical components of the aluminum slab are listed in Table 1 . This alloy was selected for its prevalence among lightweight structural alloys. The basic properties of the aluminum alloy are as follows: P-wave speed $\mathrm{Cp}=6.82 \mathrm{~km} / \mathrm{s}, \mathrm{S}$-wave speed Cs $=3.46 \mathrm{~km} / \mathrm{s}$ and mass density $\rho=2.88 \times 10^{3} \mathrm{~kg} / \mathrm{m} 3$.

Table 1. Chemical composition of the aluminum alloy slab.

\begin{tabular}{llllllll}
\hline $\mathrm{Mn}($ wt.\%) & $\mathrm{Si}($ wt. \%) & $\mathrm{Fe}($ wt.\%) & $\mathrm{Cu}($ wt.\%) & $\mathrm{Mg}($ wt.\%) & $\mathrm{Cr}(\mathrm{wt} . \%)$ & $\mathrm{Zn}(\mathrm{wt} . \%)$ & $\mathrm{Ti}(\mathrm{wt} . \%)$ \\
\hline 0.15 & 4.0 & 0.7 & 0.4 & 0.12 & 0.35 & 0.25 & 0.15 \\
\hline
\end{tabular}

\section{Ultrasonic transducers}

Broad band piezoelectric transducers (FUJI Ceramics 1045S) of diameter $20 \mathrm{~mm}$ were used because of their flat sensitivity over a wide frequency band $(200 \mathrm{kHz} \sim 1.5 \mathrm{MHz})$. The transducers allow a broad range of acoustic amplitude measurements, thus ensuring that the 
real mechanical output amplitude will be proportional to the electric amplitude excitation delivered by the power amplifier.

\section{Transmission and Acquisition}

An arbitrary waveform generator (National Instruments PXI 5105) delivered chirp signals, with frequency varying between $1 \mathrm{MHz}$ and $1.3 \mathrm{MHz}$, to the power amplifier (Ciprian USTXP-3C). The gain of the power amplifier was set at 400 with ultra-low distortion and a high-speed slew rate from $10 \mathrm{kHz}$ to $10 \mathrm{MHz}$. It allows 500 - $\mu$ s chirps to be output at incrementally increasing voltage amplitudes from $200 \mathrm{Vpp}$ to $400 \mathrm{Vpp}$.

The 4-ms received signals were synchronized to the transmission chirps and were recorded by an 8-channel 12-bits data acquisition system (National Instruments PXI 5421) at a sampling frequency of $10 \mathrm{MHz}$.

At the working frequencies, the wavelengths ranged from 3 to $7 \mathrm{~mm}$, which is much smaller than the thickness of the plate. Waves could thus be multiply reverberated in the vertical direction. Waves also reverberated in the horizontal plane, but due to the dimensions of the sample, reverberations in the horizontal plane were negligible compared to vertical reverberations. Multiple reverberations yield coda-like waveforms that look like diffuse waveforms. Hereafter, the waveforms are noted as "diffusive" even though, strictly speaking, they should be referred to as mostly multiply reverberated.

\section{Methodology and measurements}

\section{CWI analysis via amplitude-modulated diffuse ultrasound}

$\mathrm{CWI}^{20}$ was applied to quantitatively reveal the amplitude-dependent relative velocity changes $d v / v$ and decorrelation $k d$ of coda waves that can be linked to the material's nonlinearity.

Due to its robustness with respect to noise, the stretching method ${ }^{21}$ was adopted to determine $d v / v$ and $k d$. In practice, the stretching method consists of two main steps: (1) a constant change in propagation velocity between the reference signal $\left(h_{1}(t)\right)$ and the current record $\left(\mathrm{h}_{\mathrm{i}}(\mathrm{t})\right)$ is assessed, and an attempt is made to compensate it by stretching $\mathrm{h}_{\mathrm{i}}(\mathrm{t})$ along its time axis to a certain ratio $\varepsilon_{\mathrm{k}} ;(2)$ the stretched signal, i.e., $\mathrm{h}_{\mathrm{i}}\left[\mathrm{t}\left(1+\varepsilon_{\mathrm{k}}\right)\right]$ is then compared to the reference signal $\mathrm{h}_{1}(\mathrm{t})$ to detect similarities in their waveforms, which can be assessed by computing their correlation coefficient $\mathrm{CC}\left(\varepsilon_{\mathrm{k}}\right)$ within a given time window $\left[\mathrm{t}_{1} \mathrm{t}_{2}\right]$ :

$$
\mathrm{CC}\left(\varepsilon_{k}\right)=\frac{\int_{t 1}^{t 2} h_{i}\left[t\left(1+\varepsilon_{k}\right)\right] h_{1}(t) \mathrm{d} t}{\sqrt{\int_{t 1}^{t 2} h_{i}\left[t\left(1+\varepsilon_{k}\right)\right]^{2} \mathrm{~d} t \int_{t 1}^{t 2} h_{1}(t)^{2} \mathrm{~d} t}}
$$


After a grid search of $\varepsilon_{\mathrm{k}}$ over a range of plausible values, two parameters describing the change in propagation medium were obtained: the $\varepsilon_{\mathrm{k}}$ that maximizes the $\mathrm{CC}\left(\varepsilon_{\mathrm{k}}\right)$ was retained as the apparent velocity change $d v / v=\varepsilon_{\max }$; and the microstructural modification of the medium was quantified based on the residual decorrelation coefficient $\mathrm{kd}=1-\mathrm{CC}\left(\varepsilon_{\max }\right)$. It is worth to mention that the decorrelation $(\mathrm{kd})$ allows one to observe the loss of coherence in coda signals after one or several microstructural changes. Such propagation change due to local scattering change is denominated as microstructural change. The greater the kd, the larger the microstructural change(s).

With the stretching method, CWI can simultaneously reveal the time-lapse velocity change and microstructural change in the propagation medium with a high sensitivity ${ }^{2,13}$.

Without any LF pump wave, the amplitude-modulated diffuse ultrasound directly explores the so-called "quadratic hysteretic nonlinearity" by sending high-voltage amplitude diffuse waves, which are able to perturb the material elasticity. Therefore, at first order, we expect to measure the relative wave velocity change linearly proportional to the wave amplitude, meanwhile the wave amplitude increase lead to a quadratic increase in decorrelation. Below, the velocity change $(d v / v)$ (resp. microstructural change $(k d))$ and the voltage amplitude transmitted (Amp) are proposed to satisfy the following empirical law to the first order (resp. second order):

$$
\begin{gathered}
A_{d v / v}=-\frac{d v / v}{A m p} \\
A_{k d}=\frac{k d}{A m p^{2}}
\end{gathered}
$$

where $\mathrm{A}_{d v / v}$ and $\mathrm{A}_{k d}$ are the two parameters used to characterize early-stage damage in this study. It is worth to note that the transmission voltage amplitude (Amp) should be selected high enough to achieve a significant level of nonlinearity. In our case, the transmission voltage levels ranged from $200 \mathrm{Vpp}$ to $400 \mathrm{Vpp}$ are selected, which are equivalent to an acoustic strain measuring $\sim 10^{-7}$ to $\sim 10^{-5}$. 

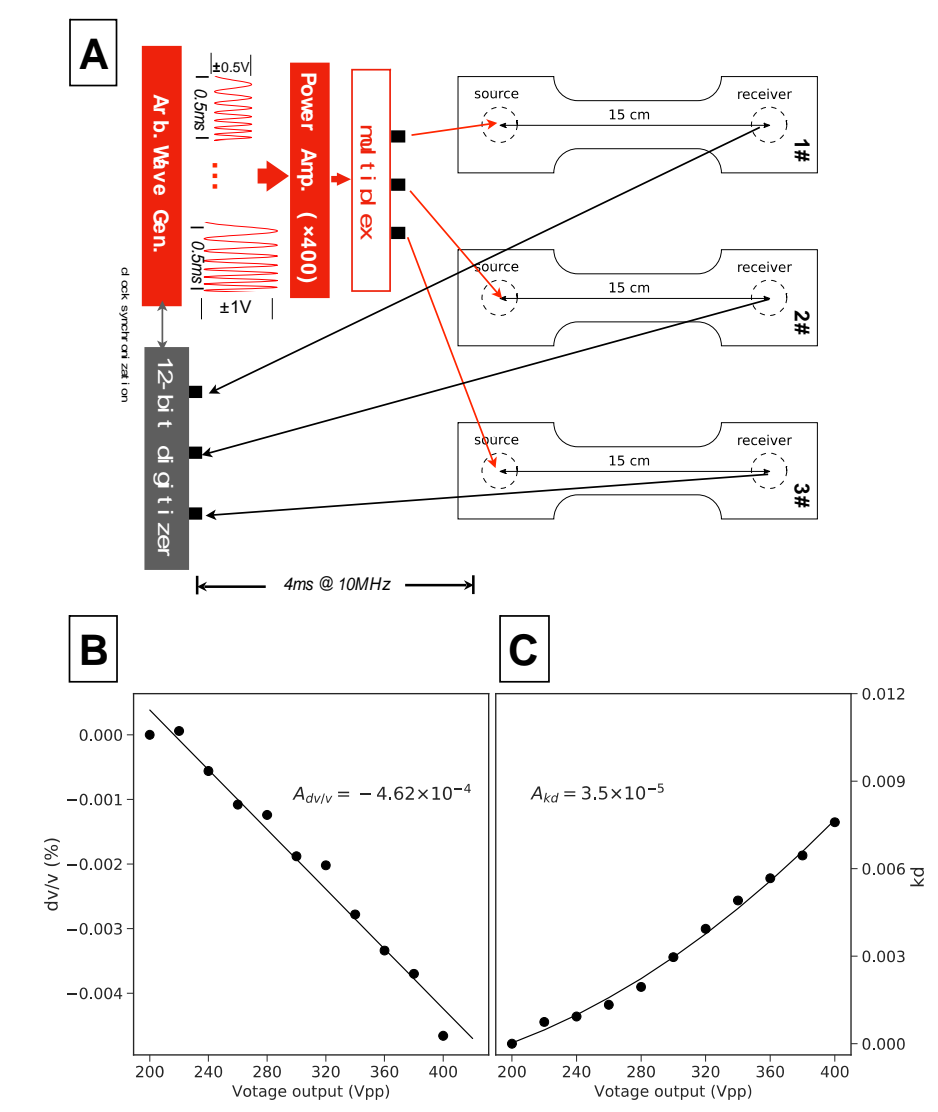

Figure 1. CWI detects alterations to steel pieces. (a) Experimental setup for
centimeter-scale specimens. Three dog-bone shaped specimens with identical
dimensions were subjected to plastic deformation at $6 \%(\# 1), 8 \%(\# 2)$ and $10 \%(\# 3)$;
(b) Apparent relative velocity change $(d v / v)$ and (c) Decorrelation $(k d)$ as a function

of the transmission voltage amplitude for specimen \#2.

For validation purposes, as an example, the method was applied to three high-manganese steel specimens with early-stage damage due to fatigue as a result of plastic deformation. These dog-bone shaped specimens were cut from an intact steel plate made of highmanganese steel. The dimensions of the specimens were identical: $34 \mathrm{~mm}$ wide, $450 \mathrm{~mm}$ long and $10 \mathrm{~mm}$ thick. To produce different levels of fatigue damage, cycles of mechanical loading were carefully performed to produce plastic deformations at $6 \%, 8 \%$ and $10 \%$ on specimen \#1, \#2 and \#3, respectively. A detailed description of the three specimens can be found in Xie et al. ${ }^{14}$. As illustrated in Fig. 1 (a), each pair of ultrasonic transducers was glued to the bottom surface of each specimen at symmetrical locations. The two transducers serve as the emitter and the receiver, and were placed $15 \mathrm{~cm}$ apart to ensure strong multiple scattering. Thanks to the use of a high-voltage multiplexer (SSM-08, Ciprian), measurements with the centimeter-scale specimens could be collected simultaneously without any unnecessary interruption. The three specimens were therefore subjected to the same experimental protocol in identical environmental conditions. By defining the lowest amplitude signal as the reference, the eleven signals acquired from each specimen as the amplitude increased from $200 \mathrm{Vpp}$ to $400 \mathrm{Vpp}$ were analyzed by CWI. The amplitude- 
dependent parameters: $\mathrm{A}_{d v / v}$ and $\mathrm{A}_{k d}$ were then measured by applying the measurement procedures proposed in Section 3.2.

Figure. 1(b) and (c) illustrate the changes to $d v / v$ and $k d$ as a function of the Amp for specimen \#2. A linear regression represents the velocity change $(d v / v)$ yields $\mathrm{A}_{d v / v}=-4.62 \times$ $10^{-4}$ per unit voltage amplitude. Simultaneously, a quadratic regression of the microstructural change $(k d)$ yields $\mathrm{A}_{k d}=3.53 \times 10^{-5}$ per unit voltage amplitude (see Eq. 2).

In Fig. 2, the nonlinear response is plotted for the three steel specimens: (a) three $\mathrm{A}_{d v / v}$ values of $-4.48 \times 10^{-4}(\# 1),-4.62 \times 10^{-4}(\# 2)$ and $-4.72 \times 10^{-4}(\# 3)$ per unit voltage amplitude were found; (b) three $\mathrm{A}_{k d}$ values of $1.31 \times 10^{-5}(\# 1), 3.53 \times 10^{-5}(\# 2)$ and $5.78 \times 10^{-5}$ (\#3) per unit excitation voltage amplitude were found.

These data demonstrate that both $\mathrm{A}_{d v / v}$ and $\mathrm{A}_{k d}$ provide a quantitative overall assessment of the specimens with different plastic deformations (despite the small deformation $\leq 10 \%$ ), from early stages of steel fatigue. Moreover, $\mathrm{A}_{k d}$ was shown to be more sensitive to the earlystage fatigue in steel than $\mathrm{A}_{d v / v}$, in line with the results observed in concrete ${ }^{9}$.

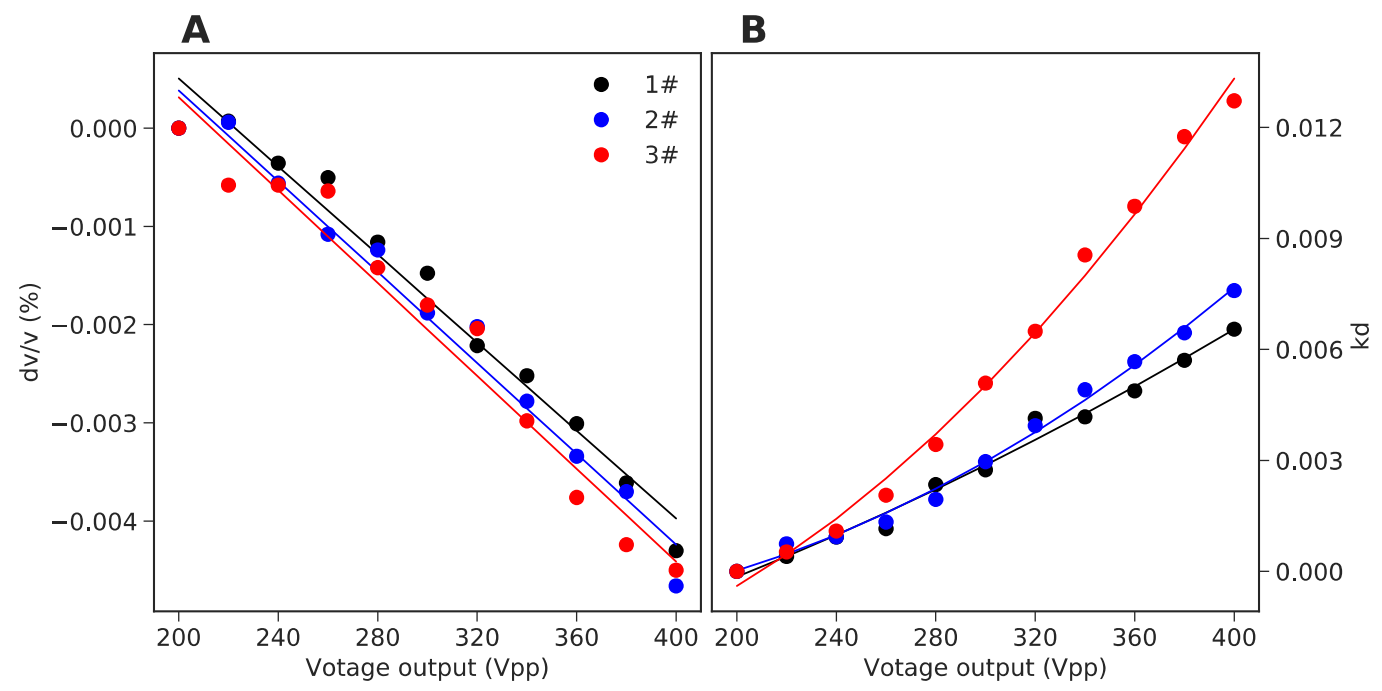

Figure 2. Early-stage damage in high-manganese steel specimens can be characterized by amplitude-modulated diffuse ultrasound (a) velocity-change parameter $A_{d v / v}$ and (b) microstructural change parameter $A_{k d}$. The second parameter $\left(A_{k d}\right)$ is more sensitive to damage than the first $\left(A_{d v / v}\right)$.

\section{Measurement procedure}

As illustrated in Fig. 3 (a), nine ultrasonic transducers were distributed over a $1 \mathrm{~m} \times 1 \mathrm{~m}$ area and glued onto the top surface of an aluminum slab to probe different regions of the specimen. Eight black transducers (labeled 1 to 8 ) served as receivers, whereas the single red transducer (labeled S) served as the emitter. 
To produce local mechanical changes induced by exposure to a high temperate, a small region (marked by the red star) on the top surface of the slab was exposed to heat-shock at $400{ }^{\circ} \mathrm{C}$ for five minutes using a high-power hot gun (1500 Watt).

The measurements were conducted by imposing transmission chirps with amplitudes increasing in $20-\mathrm{Vpp}$ steps from $200 \mathrm{Vpp}$ to $400 \mathrm{Vpp}$. The first acquisitions were taken with the intact slab 5 min before applying the heat-shock. Subsequent measurements were performed in the state 5 min after the heat-shock. It is worth to note that these two measurements only took $\sim 5$ seconds which could significantly reduce the environmental inferences to the measurements.

To make sure the microstructural alterations due to heat exposure from heat-shock was correctly localized (and to avoid possible interference of the propagation of the elastic waves from mechanical contact thermometer), we used a hand-held inferred camera (Dali TX8, $384 \times 288$ spatial resolution and $\pm 2^{\circ} \mathrm{C}$ sensitivity) for contactless temperature monitoring. GIVE THE MAX OBSERVED TEMPERATURE HERE. The finite element code COMSOL also verified the localized heat-shock processing in the slab (see the supplementary material). According to the considerable amount of published papers focusing on the microstructural evolution of 6061 aluminum alloy during heat treatment, it can be observed that the grain size increases with the increase of temperature $\left(>300^{\circ} \mathrm{C}\right)$ and time. Therefore, we will attribute the localized change in mechanical properties of the aluminum alloy to the irreversible microstructural grain growth following exposure to heat shock.

At each voltage level, the signal-to-noise ratio (SNR) was improved by repeating the chirp transmissions 100 times and averaging the corresponding received signals.

At $1 \mathrm{MHz}$, aluminum has a shear wavelength of $\sim 3 \mathrm{~mm}$ which ensures the received signals are scattered mostly by the largest grains/aggregates as well as by boundaries and multiple scattering or multiple reverberations of waves can occur ${ }^{22,23}$. Stretching analysis was performed to extract two amplitude-dependent parameters: $\mathrm{A}_{d v / v}$ and $\mathrm{A}_{k d}$ by comparing the multiple scattered responses at each voltage level with the reference response at the lowest amplitude (200 Vpp). 


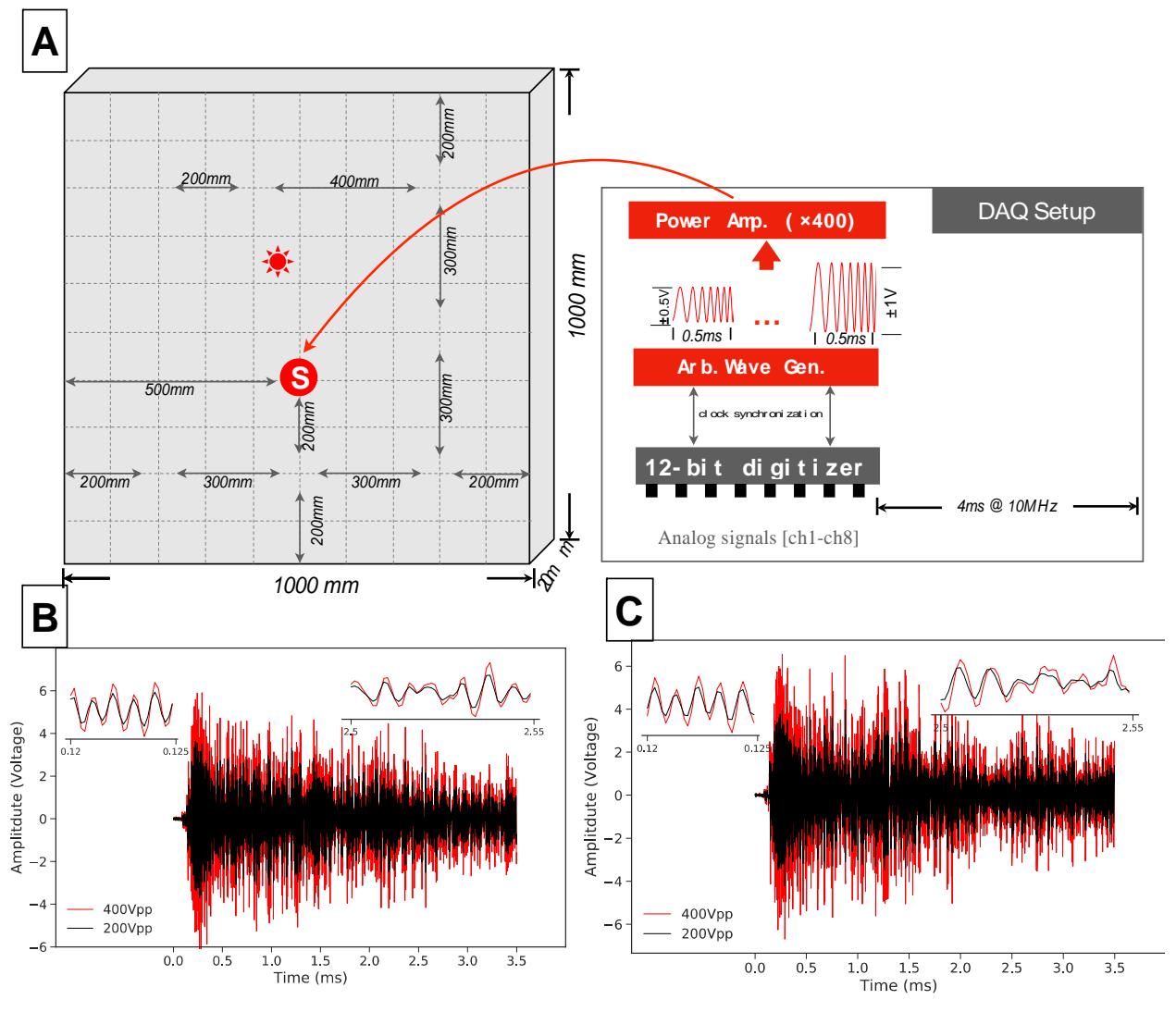

Figure 3. CWI detects mechanical change in a meter-scale aluminum slab. (a) Sample geometry, data acquisition system and transducers setup for a $20 \mathrm{~mm}$ thick meter-scale aluminum alloy slab. The black transducers labeled 1 to 8 are receivers, and the red transducer is the source. The surface of the plate, in the region marked by the red star, was heated to $400 \mathrm{oC}$. Diffuse waveforms recorded from receiver \#8 in the intact state (b) and after $400 \mathrm{oC}$ heat-shock (c). The black lines were recorded at $200 \mathrm{Vpp}$; the red lines were recorded at $400 \mathrm{Vpp}$. The insets show the details of the waveforms around the first waves detected (top left inset) and in the late coda (top right inset). The time window for CWI analysis is indicated by the gray zone on the graph.

\section{Results and discussion}

The experiment was performed in a regular office space and monitored using the procedures presented in Section 3.2. The received diffuse-like waveforms at voltage levels of $200 \mathrm{Vpp}$ and $400 \mathrm{Vpp}$ at transducer \#8 (SR8 couple) were recorded in the initial intact state (Fig. 3 (b)) and in the damaged state (Fig. 3 (c)). The two waveforms were almost identical within the early time window (top left inset of Fig. 3(b)-(c)). In contrast, compared to the intact state (top right inset of Fig. 3(b)), a more distinct phase shift in combination with waveform dissimilarity was clearly observed in the much later time window in the state after heat-shock (top right inset of Fig. 3 (c)). This observation demonstrates a more significant apparent relative velocity and waveform dissimilarity upon high-level excitation following exposure to high-temperature, with the effect probably related to the local mechanical change created between the two states. 
CWI was then applied using the stretching method to quantify changes in apparent relative velocity and waveform dissimilarities, as a function of the source amplitude level. We compared eleven diffuse signals produced by incremental amplitudes to the signal obtained with the lowest source amplitude (200 Vpp). To enhance the stability of the cross-correlation coefficient measurements, we selected a broad time window between 1 and $3.5 \mathrm{~ms}$.

Figures 4 and 5 show the apparent relative velocity changes $(d v / v)$ and decorrelations $(k d)$ as a function of the source amplitude measured at eight transducers in the intact state (black dots) and the state after exposure to a $400{ }^{\circ} \mathrm{C}$ heat-shock (red dots).

We also compared the results (See Supplementary material) due to three different time windows including an earlier (0.5-2 ms), later (2-4 ms) and narrower (1.5-3 ms) one. Despite the change in time window, the results are in good agreement with the broad window we use (1-3.5 ms) which suggests that the result is (at first order at least) independent of the time window, and that a broader time window increases the measurement stability to characterize mechanical changes.

The relative velocity change was directly proportional to the excitation amplitudes, a result that has been observed in polymethyl methacrylate (PMMA) and aluminum alloy using dynamic acousto-elastic testing ${ }^{24}$. For the specimen at pre-heat-shock, the relative velocity change was $10^{-5}$ per volt applied, which is half that of the specimen after heat-shock. These results are consistent with those obtained in previous studies ${ }^{12,25}$.

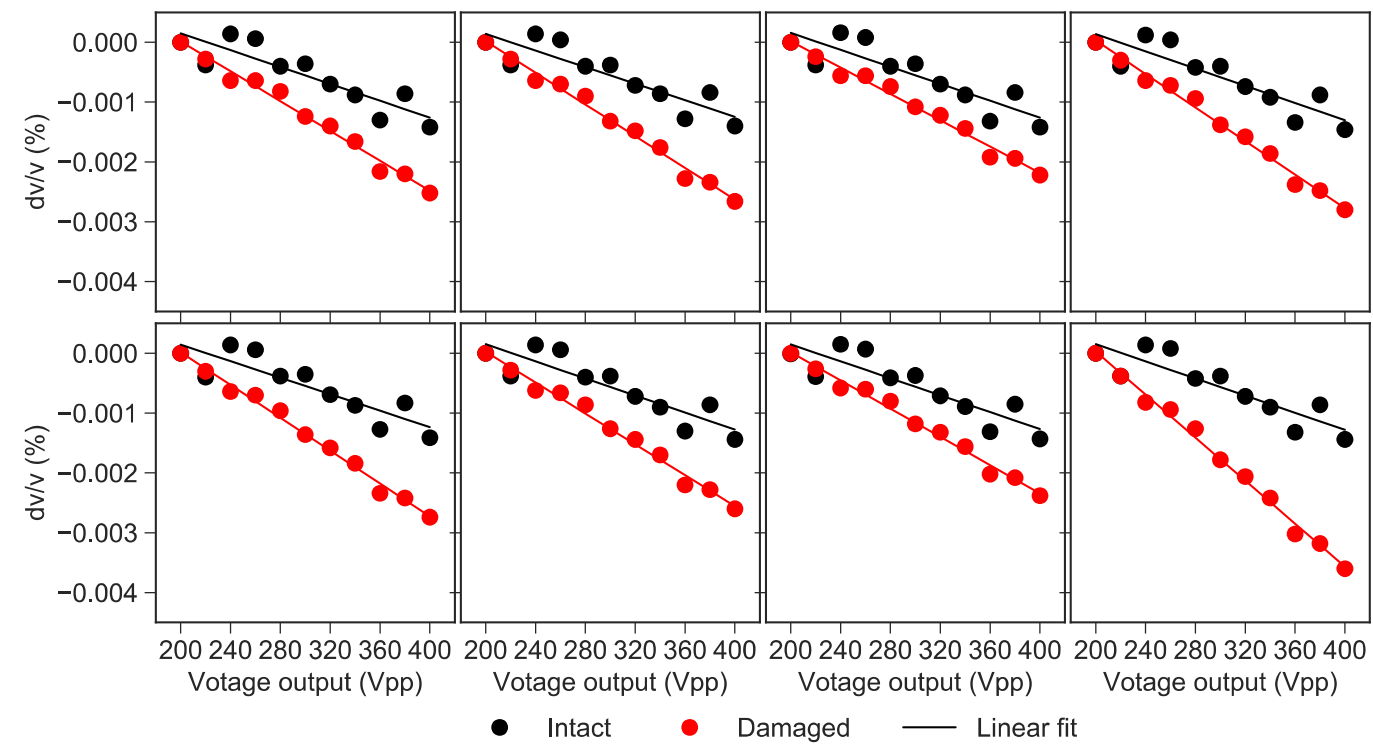

Figure 4. CWI can distinguish between intact and after heat shock aluminum based on apparent relative velocity changes $(\mathrm{dv} / \mathrm{v})$. Eight transducers placed on the aluminum alloy slab were used to acquire $\mathrm{dv} / \mathrm{v}$ in its intact state (black dots) and damaged state following $400 \mathrm{oC}$ thermal shocking (red dots). Measurements are plotted as a function of the source amplitude (Vpp). 


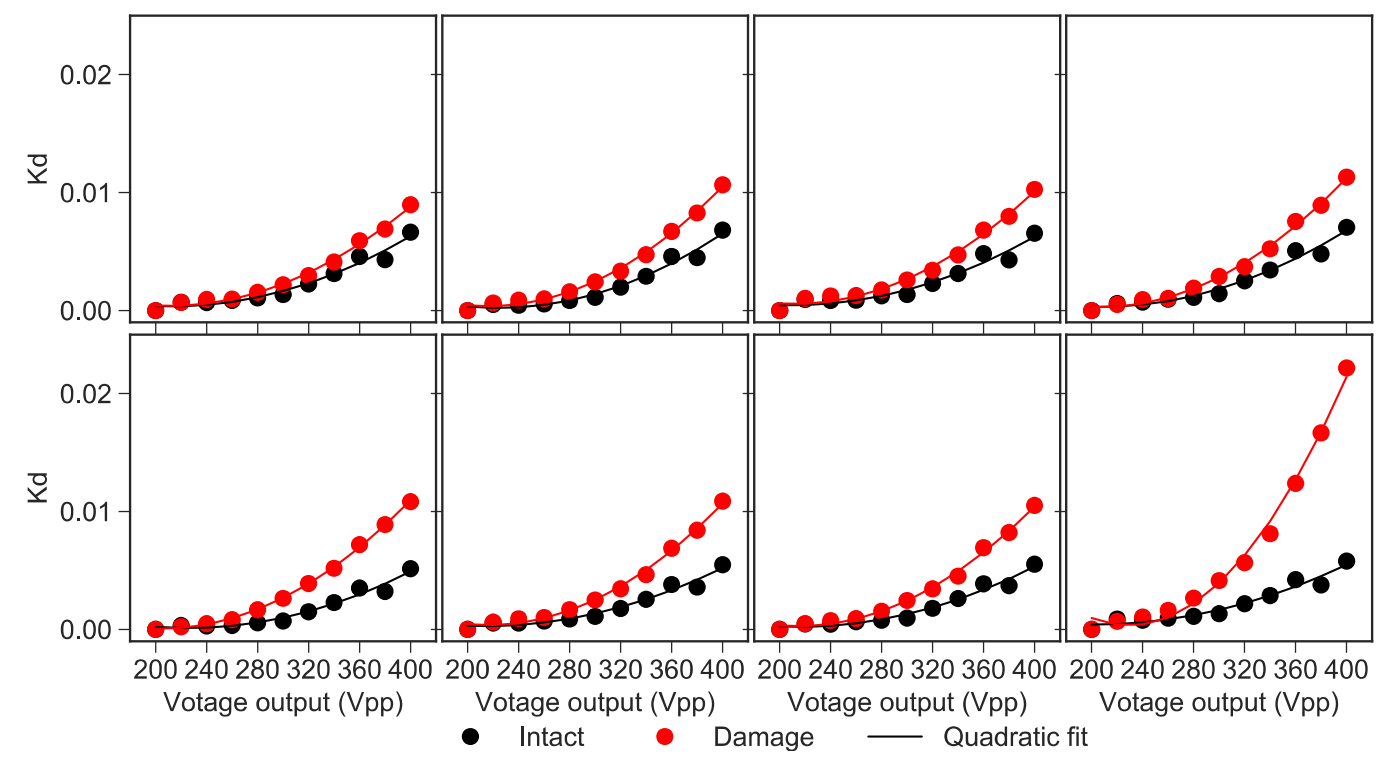

Figure 5. CWI can distinguish between intact and after heat shock aluminum based on decorrelations (kd). Eight transducers placed on the aluminum alloy slab were used to acquire kd for the intact slab (black dots) and following $400{ }^{\circ} \mathrm{C}$ thermal shocking (red dots). Data are plotted as a function of the source amplitude (Vpp).

To allow comparison, $\mathrm{A}_{d v / v}$ and $\mathrm{A}_{k d}$ acquired at each source-receiver couple before and after damage are plotted in Fig. 6 . The intact specimen shows little variance of either $\mathrm{A}_{d v / v}$ (dark black bars) or $\mathrm{A}_{k d}$ (dark red bars) across all eight source-receiver pairs. In contrast, a significant increase in both $\mathrm{A}_{d v / v}$ (light black bars) and $\mathrm{A}_{k d}$ (light red bars) was observed for all source-receiver pairs after the heat- induced mechanical change. Moreover, the highest $\mathrm{A}_{d v / v}$ and $\mathrm{A}_{k d}$ values ( 2/3 times higher than the other source-receiver couples) were found for the SR8 couple, which is positioned directly symmetrical to the heat- induced change. In the presence of local change, the $\mathrm{A}_{d v / v}$ and $\mathrm{A}_{k d}$ values were more variable than in the preheat-shock state. The significant variance can mainly be attributed to the uneven distribution of diffuse wave sensitivities, which depend on the locations of the transducers. Similar to the work of Pacheco and Snieder ${ }^{26}$ and Larose et al ${ }^{27}$, we can identify sensitivity kernels based on the propagation of the intensity within the plate. Contrary to the diffusion equation, which holds in multiple scattering media, for this system we must take into account multiple reverberation of the energy on the vertical axes, and on horizontal axes. This reverberation can be numerically calculated and will be the subject of further investigations. For the moment, we can only hypothesize, since the highest value for $\mathrm{A}_{k d}$ was observed for the closest source-change-receiver combination, that an inverse problem based on proper sensitivity kernels can locate the change. 


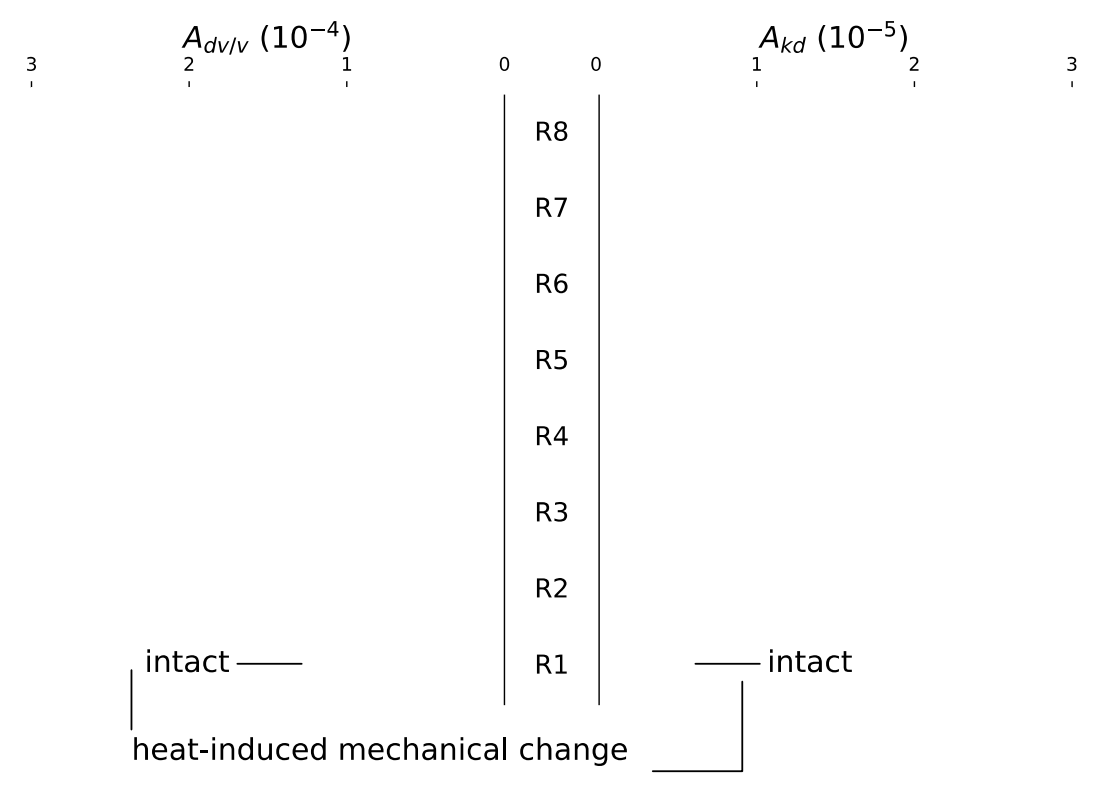

Figure 6. $\mathrm{A}_{d v / v}$ and $\mathrm{A}_{k d}$ acquired at the eight transducers for the intact aluminum plate (dark bars) and following heatinduced mechanical change (light bars).

The results obtained from amplitude-dependent diffuse-like waves raise another question: why are both $\mathrm{A}_{d v / v}$ and $\mathrm{A}_{k d}$ sensitive to the microstructural alteration exposed to heat-shock in the test specimens?

To address this question, we note that the increase in $\mathrm{A}_{d v / v}$ and $\mathrm{A}_{k d}$ with the source amplitude level is very similar to the amplitude dependency observed in strong heterogeneous media using low frequency (LF) pump waves ${ }^{9,12}$. For example, CWI at several hundred $\mathrm{kHz}$ has been used as probe wave with an amplitude- modulated frequency-swept pump wave at a few $\mathrm{kHz}$ to detect millimeter-scale cracks in homogeneous solids. For these types of medium, various possible explanations were proposed based on previous studies ${ }^{7,28}$. These explanations are compatible with the widely accepted theory of mesoscopic nonlinearity, which states that the nonlinear response depends on the presence of micro defects or soft inhomogeneities that act as nonlinear sources altering the overall level of the material's nonlinearity. This type of nonlinear behavior reduces (softens) the resonance frequency. The softening of the resonance frequency can be directly compared to the CWI parameter $d v / v$, which is directly proportional to the LF pump wave amplitude. The quadratic increase in $\mathrm{A}_{k d}$ is more complex to explain and will require further investigation.

Even at low excitation levels $\left(10^{-7}\right)$, the hysteretic nonlinearity in metals has been widely documented $^{1,4,25,29}$.

It is worth noting that changes in weak contacts of the microstructural alteration at the grain scale are thought to be responsible for the main contribution, especially for HF diffuse wave 
acoustic energy transmission (excitation voltage amplitude). The opening or closing of weak contacts due to the acoustic energies $\left(10^{-7} \sim 10^{-5}\right)$ of the passing waves may cause dynamic switching of the reverberation paths. This phenomenon could explain the nonlinear behavior observed in the weakly heterogeneous metal specimens tested here. Although the acoustic strains induced by amplitude-modulated waves are 1-2 orders of magnitude smaller than those induced by LF pump waves, this hypothesis is in agreement with the behavior of the pump wave in heterogeneous media. Additional insights may be obtained by observing micro defects using diffuse waves at millimeter-scale wavelengths. Numerical study ${ }^{30}$ has shown that the long propagation paths of diffuse waves in a medium can enhance localized spatial resolution of the changes at its wavelength $\sim 10$-fold. As a result, here, we can detect the micro defects induced at a distance of several hundreds or dozen of microns.

In principle, modifications to the acoustic properties of the waveform following strong excitation by HF-amplitude-modulated diffuse-like waves should be particularly sensitive to weak contact changes at grain scales induced by heat-shock. This was confirmed by the greater sensitivity of $\mathrm{A}_{k d}$ than $\mathrm{A}_{d v / v}$ to both early fatigue damage in steels and heat-induced mechanical change in the aluminum alloy slab. However, the physics behind these nonlinear theories in metals is an ongoing research topic.

\section{Conclusion}

In this study, we used a novel method based on HF diffuse ultrasound at successive excitation amplitudes to characterize localized mechanical change induced by a heat-shock in a meterscale aluminum alloy slab. The major findings were:

(1) using CWI, a linear decrease in $d v / v$ (relative velocity change) and a quadratic increase in $k d$ (decorrelation) as a function of increasing source amplitude was observed. Thus, the parameters $\mathrm{A}_{d v / v}$ and $\mathrm{A}_{k d}$ are sensitive to localized mechanical properties to microstructural alterations.

(2) overall fatigue damage caused by plastic deformations varying from $6 \%$ to $10 \%$ in centimeter-scale high-manganese steel specimens, and instantaneous $400{ }^{\circ} \mathrm{C}$ heat-induced local mechanical change in meter-scale aluminum plate were successfully characterized by the $\mathrm{A}_{d v / v}$ and $\mathrm{A}_{k d}$ parameters.

(3) the observed amplitude dependence of HF ultrasound may be caused by nonlinear hysteresis mechanisms, and the observed spatial sensitivity of $\mathrm{A}_{d v / v}$ and $\mathrm{A}_{k d}$ could form the basis of an inverse problem for future imaging techniques. 
The major advantages of the method used here over conventional approaches include: 1) significant reduction of the inspection time by comparing to the conventional monitoringoriented CWI method thanks to a simple and robust experimental setup; 2) high sensitivity to the material status in the early stages of damage, and to mechanical change; 3) compatibility between laboratory-based and on-site measurements; 4) with appropriate spatial sensitivity kernels, the method can not only be used to assess damage levels but also has great potential to image the material's status in the early stages of damage.

\section{Disclosure statement}

The author(s) have no potential conflicts of interest to declare with respect to the research, authorship, and/or publication of this article.

\section{Funding}

The authors acknowledge funding from the National Natural Science Foundation of China (Grant number:41874061,51738001,51779061), Public Science and Technology Research Funds Project of Ministry of Land and Resources of China (201511037-02), from Labex OSUG@2020 and from the University Grenoble Alpes IDEX.

\section{References}


1. Zeiger A and Jassby K. Measurement of acoustoelastic coefficients of rayleigh waves in steel alloys. Journal of Nondestructive Evaluation 1982; 3(2): 115-124.

2. Larose $\mathrm{E}$ and Hall S. Monitoring stress related velocity variation in concrete with a $2 \times 10$ 5 relative resolution using diffuse ultrasound. The Journal of the Acoustical Society of America 2009; 125(4): 1853-1856.

3. Payan C, Garnier V, Moysan J et al. Determination of third order elastic constants in a complex solid applying coda wave interferometry. Applied Physics Letters 2009; 94(1): 011904.

4. Johnson $P$ and Sutin A. Slow dynamics and anomalous nonlinear fast dynamics in diverse solids. The Journal of the Acoustical Society of America 2005; 117(1): 124-130.

5. Tremblay N, Larose E and Rossetto V. Probing slow dynamics of consolidated granular multicomposite materials by diffuse acoustic wave spectroscopy. The Journal of the Acoustical Society of America 2010; 127(3): 1239-1243.

6. Ulrich TJ, McCall K and Guyer R. Determination of elastic moduli of rock samples using resonant ultrasound spectroscopy. The Journal of the Acoustical Society of America 2002; 111(4): 1667-1674.

7. Riviere J, Renaud G, Guyer RA et al. Pump and probe waves in dynamic acousto-elasticity: Comprehensive description and comparison with nonlinear elastic theories. Journal of Applied Physics 2013; 114(5): 054905.

8. Hilloulin B, Zhang Y, Abraham O et al. Small crack detection in cementitious materials using nonlinear coda wave modulation. Ndt \& E International 2014; 68(68): 98-104.

9. Zhang Y, Tournat V, Abraham $\mathrm{O}$ et al. Nonlinear coda wave interferometry for the global evaluation of damage levels in complex solids. Ultrasonics 2017; 73: 245-252. 10. Guyer RA and Johnson PA. Nonlinear mesoscopic elasticity: Evidence for a new class of materials. Physics Today 1999; 52(4): 30-36.

11. Solodov IY, Krohn N and Busse G. Can: an example of nonclassical acoustic nonlinearity in solids. Ultrasonics 2002; 40(1): 621-625.

12. Shokouhi P, Rivière J, Lake CR et al. Dynamic acousto-elastic testing of concrete with a coda-wave probe: Comparison with standard linear and nonlinear ultrasonic techniques.

Ultrasonics 2017.

13. Planès T and Larose E. A review of ultrasonic coda wave interferometry in concrete. Cem Concr Res 2013; 53: 248-255.

14. Xie F, Li W and Zhang Y. Monitoring of environmental loading effect on the steel with different plastic deformation by diffuse ultrasound. Structural Health

Monitoring-an International Journal 2019; 18(2): 602-609. 
15. Zhang Y, Tournat V, Abraham $\mathrm{O}$ et al. Nonlinear mixing of ultrasonic coda waves with lower frequency-swept pump waves for a global detection of defects in multiple scattering media. Journal of Applied Physics 2013; 113(6): 064905.

16. Legland J, Zhang Y, Abraham $\mathrm{O}$ et al. Evaluation of crack status in a meter-size concrete structure using the ultrasonic nonlinear coda wave interferometry. Journal of the Acoustical Society of America 2017; 142(4): 2233-2241.

17. Maljaars JJ, Soetens FF and Katgerman L. Constitutive model for aluminum alloys exposed to fire conditions. Metallurgical and Materials Transactions A-physical Metallurgy and Materials Science 2008; 39(4): 778-789.

18. Summers P, Matulich R, Case SW et al. Post-fire mechanical properties and hardness of 5083 and 6082 aluminum alloys. In ASME 2012 International Mechanical Engineering Congress and Exposition. American Society of Mechanical Engineers, pp. 1251-1259.

19. Summers PT, Chen Y, Rippe CM et al. Overview of aluminum alloy mechanical properties during and after fires. Fire Science Reviews 2015; 4(1): 3.

20. Snieder R, Grêt A, Douma H et al. Coda wave interferometry for estimating nonlinear behavior in seismic velocity. Science 2002; 295(5563): 2253-2255.

21. Hadziioannou C, Larose E, Coutant O et al. Stability of monitoring weak changes in multiply scattering media with ambient noise correlation: Laboratory experiments. The Journal of the Acoustical Society of America 2009; 125(6): 3688-3695.

22. Weaver RL. On diffuse waves in solid media. Journal of the Acoustical Society of America 1982; 71(6): 1608-1609.

23. Weaver RL and Lobkis OI. Temperature dependence of diffuse field phase.

Ultrasonics 2000; 38(1): 491-494.

24. Renaud G, Talmant M, Callé S et al. Nonlinear elastodynamics in microinhomogeneous solids observed by head-wave based dynamic acoustoelastic testing. The Journal of the Acoustical Society of America 2011; 130(6): 3583-3589.

25. Riviere J, Remillieux MC, Ohara Y et al. Dynamic acousto-elasticity in a fatigue- cracked sample. Journal of Nondestructive Evaluation 2014; 33(2): 216-225.

26. Pacheco $\mathrm{C}$ and Snieder R. Time-lapse travel time change of multiply scattered acoustic waves. Journal of the Acoustical Society of America 2005; 118(3): 13001310.

27. Larose E, Planes T, Rossetto V et al. Locatingasmallchangeinamultiplescattering environment. Applied Physics Letters 2010; 96(20): 204101.

28. Tournat V and Gusev V. Nonlinear effects for coda-type elastic waves in stressed granular media. Physical Review E 2009; 80(1): 011306. 
29. Nagy PB. Fatigue damage assessment by nonlinear ultrasonic materials characterization. Ultrasonics 1998; 36(1-5): 375-381.

30. Xie F, Moreau L, Zhang Y et al. A bayesian approach for high resolution imaging of small changes in multiple scattering media. Ultrasonics 2016; 64: 106-114.

${ }^{1}$ Institute of Geophysics, China Earthquake Administration, Beijing 100081, China

${ }^{2}$ Harbin Engineering University, Harbin 150001, China

${ }^{3}$ ISTerre, Universite` de Grenoble Alpes, CNRS, Grenoble, France

${ }^{4}$ Laboratoired'Acoustiquedel'Universite 'duMans, LAUM-UMRCNRS6613,

LeMansUniversite', Avenue Olivier Messiaen, 72085 LE MANS CEDEX 9, France

${ }^{5}$ College of Architecture and Civil Engineering, Beijing University of Technology, Beijing 100022, China

${ }^{6}$ School of Microelectronics, Xidian University, Xi'an ,710071, China

Corresponding author:

Yuxiang Zhang, Harbin Engineering University, Harbin 150001, China. Email:

yuxiang.zhang@outlook.com 